easily get their bearings by feeling the direction of the leaves." But the first occasion on which it was brought under the notice of scientific men appears to have been in communications addressed to the National Institute at Washington in August, 1842, and January, 1843, by General Alvord. The accuracy of his statement having been questioned, the General presented another communication at the second meeting of the American Association for the Advancement of Science, held at Cambridge, Mass., in August, 1849, in which he confirms his own observations by those of other officers, all agreeing in the conclusion that the radical leaves of the plant really present their edges north and south, while their faces are turned east and west, the leaves on the developed stems of the flowering plant, however, taking rather an intermediate position between their normal or symmetrical arrangement and their peculiar meridional position. For the following particulars respecting the phenomena exhibited by the compass-plant we are indebted mainly to a paper by Mr. W. F. Whitney, read before the Harvard Natural History Society and printed in the American

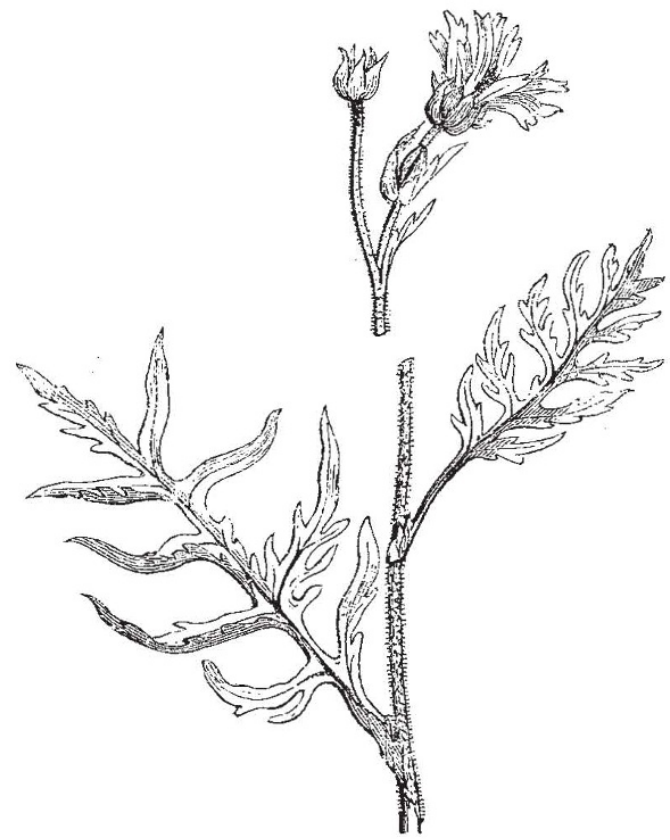

The Compass-plant (Silphium laciniatum)

Naturalist for March, I87 I, and to some subsequent notes by Prof. Asa Gray and Mr. Thos. Meehan.

The cause of the ordinary position of the leaves of most plants, one surface being directed towards the sky and the other towards the earth, is generally believed to be a difference in the sensitiveness to light of the two surfaces, the epidermal tissue of the upper being generally denser and less pervious to light than that of the under surface. It is possible also that something may be due to the fact that the under surface of the leaf is almost always more copiously furnished with stomates or "breathing-pores," as they are often inaccurately termed, minute orifices, which serve to promote a diffusion of gases between the external air and the intercellular cavities within the tissue, and especially an abundant exhalation of aqueous vapour. A microscopical examination of the leaves of the compass-plant shows that the structure of the epidermal tissue of the two surfaces is similar, and also that the number of stomates in each corresponds, affording in this respect a contrast to other allied species of the genus Silphium, which do not exhibit the phenomenon of polarity, and in whose leaves the stomates were found to be from two to three times as numerous on the under as on the upper surface. If, therefore, the object to be gained is an equal sensitiveness to light, it is obvious that the two surfaces will receive an equal mean amount of light during the twentyfour hours, if they face the east and the west, rather than if they face the north and the south, or the earth and the sky. An attempt has also been made to explain the phenomenon of polarity by currents of electricity induced by the peculiar chemical composition of the substances secreted by the stem and the leaves, but not with much success.

In a recent communication to the Academy of Natural Sciences at Philadelphia, Mr. Meehan says that those who affirm that the leaves are directed to the north, and those who say that there is no such tendency, are both right. He watched a plant in his own garden, and observed the unmistakable northern tendency in the leaves when they first came up, and until they were large and heavy, when winds and rain bore them in different directions, and they evidently had not the power of regaining the points lost. It would appear, therefore, to depend on the season when the observation is made whether the leaves are seen to bear northwards or not.

The same observer records also the following facts with regard to the flowers of the compass-plant. The expansion of the ray-florets in August was observed to begin at daybreak, 4 A.M. ; forty minutes afterwards the florets of the disc began to open, and the whole of the corollas were expanded in about three-quarters of an hour, after which there was no further growth in the corollas. The stamens and pistils were gradually protruded beyond the corolla, and the lengthening of the stamens ceased at 6 A.M. After 6.20 no further growth was noticed in the flowers. Later on they were visited by insects, causing the detachment of the florets of the disc, and the scattering of the pollen on to the ray-florets, which were thus fertilised. There appear, in fact, to be three phases of growth, with a slight rest between each, the pistil taking the most time, then the stamens, and the corolla the least; but the whole growth of the day is included within two hours.

The geographical range of the plant is stated to be from Texas on the south to Iowa on the north, and from Southern Michigan on the east to 300 or 400 miles west of Missouri and Arkansas. Its chief habitat is rich prairie land. Our illustration is taken partly from the plate in Jacquin's "Eclogæ," the only good drawing of the plant published, assisted by comparison with dried specimens in the Kew Herbarium.

A. W. B.

\section{EXPERIMENTS WITH THE RADIOMETER}

II.

HAVING ascertained that the action of the radioHeter was due to the internal movement of the molecules of the residual gas, it became important to obtain as much information as possible respecting the physical properties of this residue.

In the apparatus constructed for this purpose a vertical plate is suspended by a glass fibre, which it twists in opposite directions alternately, instead of continuously rotating in one direction, as in the ordinary radiometer. Attached to this apparatus there is :- $a$, a sprengel pump ; $b$, an arrangement for producing a chemical vacuum; $c$, a lamp with scale, on which to observe the luminous index reflected from a mirror; $d$, a standard candle at a fixed distance; and $e$, a small vacuum tube, with the internal ends of the platinum wires close together. I can therefore take observations of-

I. The logarithmic decrement of the arc of oscillation when under no influence of radiation.

\footnotetext{
1 Continued from p. 227.
} 
2. The successive swings and final deflection when a candle shines on one end of the blackened bar.

3. The appearance of the induction spark between the piatinum wires.

I measures the viscosity of the gas ; 2 enables me to calculate the force of radiation of the candle; and 3 enables me to form an idea of the progress of the vacuum according as the interior of the tube becomes uniformly luminous, striated, luminous at the poles only, or black and nonconducting. The movement is started by rotating the whole apparatus through a small angle, and the observation consists in noting the successive amplitudes of vibration when the instrument is left to itself, a mirror and spot of light being employed for this purpose. The amplitudes form a decreasing series, with a regular logarithmic decrement. Up to the point at which the vacuum is apparently equal to a Torricellian vacuum, the logarithmic decrement is nearly constant; but as the exhaustion proceeds beyond this point, it becomes smaller, and the force of repulsion approaches a maximum; when the logarithmic decrement is about one fourth of what it was at the commencement, the force of repulsion begins to diminish, and at much higher exhaustions it nearly ceases.

I have experimented with different gases in the apparatus, and by means of a McLeod gauge attached to a mercury-pump, I have been able to measure the atmosoheric pressure at any desired state of exhaustion. From the results of the measurements of the force of repulsion and of the viscosity of the residual gas, 1 have plotted the observations in curves, which show how the viscosity of the residual gas is related to the force of repulsion exerted by radiation.

I have supposed my scale to be I,000 metres long, and to represent one atmosphere. Each millimetre, therefore, stands for the millionth of an atmosphere.

When the residual gas is air, the viscosity, measured by the logarithmic decrement of the arc of oscillation, is practically constant up to an exhaustion of 250 millionths of an atmosphere, or $0^{*} 19$ millim. of mercury, having only diminished from 0.126 at the normal pressure of the atmosphere, to 0.112 . It now begins to fall off and at $O$ I of a millionth of an atmosphere the logarithmic decrement has fallen to about oor. Simultaneously with this decrease in the viscosity, the force of repulsion exerted on a black surface by a standard light varies. It increases very slowly till the exhaustion has risen to about 70 millionths of an atmosphere; at about 40 millionths the force is at its maximum; and it then sinks very rapidly till at 0.1 millionth of an atmosphere it is less than one-tenth of its maximum.

When the residual gas is oxygen the logarithmic decrement is 0.126 at the atmospheric pressure, and at 2 millionths of an atmosphere it is 0.02. The force of repulsion in oxygen increases very steadily up to an exhaustion of alout 40 millionths of an atmosphere; it is at its maximum at about 39 millionths, and thence declines very rapidly.

It is not necessary to get so high an exhaustion with hydrogen as with other gases to obtain considerable repulsion. The viscosity at the normal pressure is measured by a logarithmic decrement of 0.063 ; at 50 millionths it is 0.046 , when it rapidly sinks. The force of repulsion is very great in a hydrogen vacuum being, in comparison with the maximum in an air vaçuum as 70 to $4 \mathrm{r}$.

Carbonic acid has a viscosity of about oor at the normal pressure, being between air and hydrogen, but nearer the former. The force of repulsion does not rise very high and soon falls off.

A long series of observations have been taken, at different derrees of exhaustion, on the conductivity of the residual gas to the spark from an induction coil. Working with air I find that at a pressure of about 40 millionths of an atmosphere, when the repulsive force is near its rnaximum, a spark, whose striking distance at the normal pressure is half an inch, will illuminate a tube having terminals 3 millimetres apart. When I push the haustion further the $\frac{1}{2}$-inch spark ceases to pass, but a I-inch spark will still illuminate the tube. As I get nearer to a vacuum more power is required to drive the spark through the tube, but, at the highest exhaustions, I can still get traces of conductivity when an induction coil, actuated with five Grove's cells, and capable of giving a 6-inch spark, is used.

When so powerful a spark is employed it sometimes happens that the glass is perforated, thus causing a very slight leakage of air into the apparatus. The logarithmic decrement now slowly rises, the repulsive force of the candle increases to its maximum and then slowly diminishes to zero, the logarithmic decrement continuing to rise till it shows that the internal and external pressure are identical.

In preparing experimental radiometers I prefer to exhaust direct to one or two millionths of an atmosphere. By keeping the apparatus during the exhaustion in a hot air-bath heated to about $300^{\circ} \mathrm{C}$. for some hours, the occluded gases are driven off from the interior surface of the glass and the fly of the radiometer. The whole is then allowed to cool, and attenuated air from the air-trap is put in in small quantities at a time, until the McLeod gauge shows that the best exhaustion for sensitiveness is reached; if necessary, this point is also ascertained by testing with a candle. In this manner, employing hydrogen instead of air for the gaseous residue, and using roasted mica vanes at an angle with the axis, I can get very considerably increased sensitiveness in radiometers. I am still unable, however, to get them to move in moonlight, while my sensitive torsion balance does easily.

I have tried many experiments with the view of putting the theory I have referred to in my former paper (NATURE, vol. xv. p. 224) to a decisive test. The repulsive force being due to a molecular disturbance causing a reaction between the fly and the glass case of a radiometer, it follows that, other things being equal, the fly should revolve faster in a small bulb than in a large one. I therefore constructed a double radiometer which shows this fact in a very satisfactory manner. It consists of two bulbs, one large and the other small, blown together so as to have a wide passage between them. In the centre of each bulb is a cup, held in its place by a glass rod, and in the bulbs is a small fourarmed fly with roasted mica discs blacked on one side. The fly can be balanced on either cup. In the smaller bulb there is about a quarter of an inch between the vanes and the glass, whilst in the larger cup there is a space of half an inch. The mean of several experiments shows that in the small bulb the fly rotates about 50 per cent. faster than in the large bulb when exposed to the same source of light.

One of the arms of another radiometer was furnished with roasted mica discs blacked on alternate sides. The other arm was furnished with clear mica discs. The two arms were pivoted independently of each other, and one of them was furnished with a minute fragment of iron, so that by means of a magnet I could bring the arms in contact, the black surface of the mica then having a clear plate of mica in front of it. On bringing a lighted candle near the instrument and allowing it to shine through the clear plate, on the blackened mica, the clear plate is at once driven away, till the arm sets at right angles to the other.

Two currents of force, acting in opposite directions, can exist in the same bulb. I have prepared a double radiometer in which two flys are pivoted one over the other, and having their blackened sides turned in opposite directions. On bringing a lighted candle near the flys rotate in opposite directions.

Experiment shows that the molecular disturbance 
which constitutes the force can be reflected from a plane surface in such a manner as to change its direction. A two-disc radiometer was made having flat opaque mica discs blacked on one side. In front of the black surface of the mica and about a millimetre off, is fixed a larger disc of thin clear mica. On bringing a candle near, the molecular pressure streaming from the black surface is caught by the clear plate and thrown back again, causing pressure behind instead of in front, and the result is rapid rotation in the negative direction, the black side now moving towards the light.

The above actions can be explained on the "evaporation and condensation" theory, as well as by that of molecular movement. I therefore devised the following test to decide between these two theories. A radiometer has its four discs cut of very clear and thin plates of mica and these are mounted in a somewhat large bulb. At the side of the bulb in a vertical plane, a plate of mica, blacked on one side, is fastened in such a position that each clear vane in rotating shall pass it, leaving a space between of about a millimetre. If a candle is brougiat near, and by means of a shade the light is allowed to fall only on the clear vanes, no motion is produced; but if the light shines on the black plate the fly instantly rotates as if a wind were issuing from this surface, and keeps on moving as long as the light is near. This could not happen on the evaporation and condensation theory, as this requires that the light should shine intermittently on the black surface in order to keep up continuous movement.

The experiments with the double radiometer of different sizes showed that the nearer the absorbing surface was to the glass the greater was the pressure produced. To test this point in a more accurate manner a torsion balance was fitted up with a glass suspending fibre and a reflecting mirror. At one end of the beam is a disc of roasted mica blacked on one side. In front of this black surface, and parallel to it, is a plate of clear mica so arranged that its distance from the black surface can be altered as desired at any degree of exhaustion without interfering with the vacuum. This apparatus has proved that when light falls on the black surface molecular pressure is set up, whatever be the degree of exhaustion. A large series of observations have been taken with it with the result of not only supplying important data for future consideration, but of clearing up many anomalies which were noticed, and of correcting many errors into which $I$ was led at earlier stages of the research. Among the latter may be mentioned the speculations in which I indulged as to the pressure of sunlight on the earth.

I now tried similar experiments to the above, using the best conductors of heat instead of the worst. A radiometer, the fly of which is made of metallic plates perfectly flat and lampblacked on one side, is much less sensitive to light than one of mica or pith; but, as I proved in a paper sent to the Royal Society in January last year, it is more sensitive to dark heat, which indeed causes the black face of a metal radiometer rapidly to rotate in a negative direction, the black continuing to advance until the temperature has become uniform throughout; but as soon as the source of heat is removed the fly commences to revolve with rapidity the positive way, the black retreating as it would if light shone on it.

Experiments with discs of aluminium, mounted diamondwise and turned up and folded at different angles, show that shape has even a stronger influence than colour. A convex bright surface is strongly repelled, whilst a concave black surface is not only not repelled by radiation, but is actually attracted.

Carefully-shaped cups of gold, aluminium, and other metals, have been tried, as well as cones of the same materials. If a two-disc, cup-shaped radiometer, facing opposite ways and both sides bright, is exposed to a standard candle 3.5 inches off, the fly rotates continuously at the rate of one revolution in 3.37 seconds. A screen placed in front of the concave side so as to let the light shine only on the convex surface, the latter is repelled, causing continuous rotation at the rate of one revolution in 7.5 seconds. When the convex side is screened off so as to let the light strike only on the concave side, contmuous rotation is produced at the rate of one revolution in 6.95 seconds, the concave side being attracted.

These experiments show that the repulsive action of radiation on the convex side is about equal to the attractive action gradation on the concave side, and that the double speed with which the fly moves when no screen is interposed is the sum of the attractive and repulsive actions.

With a two-disc, cup-shaped aluminium radiometer as above, lampblacked on the concave surfaces, the action of light is reversed, rotation taking place, the bright convex side being repelled and the black concave attracted. When the light shines only on the bright convex side no movement is produced, but when it shines only on the black concave side this is attracted, producing rotation.

Light shining on a cup-shaped radiometer similar to the above, but having the convex side black and the concave bright, causes it to rotate rapidly, the convex black being repelled. No movement is produced on letting the light shine on the bright concave surface, but good rotation is produced when only the black convex surface is illuminated.

With a cup-shaped radiometer like the above, but blacked on both sides, a candle causes rapid rotation, the convex side being repelled. On shading off the light from the concave side the rotation continues, but much more slowly; on shading off the convex side the concave is strongly attracted, causing rotation.

Radiometers have also been made with cups and cones of plain mica, roasted mica, pith, paper, \&c., and they have been made either plain or blacked on one or both surfaces. These have also been balanced against each other, and against metal plates, cups, and cones. The results are of considerable interest but too complicated to explain without numerous diagrams. The broad facts are contained in the above selections from my experiments.

Some of the phenomena produced by the action of light on the cup-shaped vanes of a radiometer may be explained on the assumption that the molecular pressure acts chiefly in a direction normal to the surface of the vanes. A convex surface would therefore cause greater pressure to be exerted between itself and the inner surface of glass than could a concave surface. But it is not easy to see how such an hypothesis can explain the behaviour of those instruments where the action of the bright convex surface more than overcomes the superior absorptive and radiating power of the concave black surface ; and the explanation appears to fail to account for the powerful attraction which a lighted candle is seen to exert on the concave surfaces in other instruments.

These experiments, interpreted by the light of the dynamical theory given in my last communication, explain very clearly how it was that I obtained such strong actions in my earlier experiments when using white pith, and employing the finger as a source of heat; and how it happened that I did not discover for some time that dark heat and the luminous rays were essentially different in their actions on black and white surfaces. Rays of high intensity pass through the glass bulb without warming it ; they then, falling on the white surface, are simply reflected off again; but, falling on the black surface, they are absorbed, and raising its temperature, produce the molecular disturbance which causes motion. Rays of low intensity, however, do not pass through the glass to any great extent, but being absorbed, raise its temperature. This warmed spot of glass now becomes the repelling body through the intervention of the mole- 
cules rebounding from it with a greater velocity than that at which they struck it. The molecular pressure, therefore, in this case streams from the inner surface of the warm spot of glass on which the heat rays have fallen, and repels whatever happens to be in front of it, quite irrespective of the colour of its surface.

\section{WilliaM CROOKES}

\section{THE SPONTANEOUS GENERATION QUESTION}

TWO contributions have recently been made to this subject through the Royal Society by Dr. W. Roberts and Prof. Tyndall. Dr. Roberts's communication is as follows ${ }^{1}$ :-

In a recent communication to the Royal Society, Dr. Bastian ${ }^{2}$ brought forward some experiments to show that while an ecic urine usually remains barren after being boiled a few minutes, the same urine becomes fertile when similarly treated, if previously neutralised or rendered alkaline by liquor potassae, especially if it be afterwards maintained at a temperature of $115^{\circ} \mathrm{F}$. or $122^{\circ} \mathrm{F}$. In this respect urine only conforms to the genexal rule observed by myself and formulated in my previous communication to the Society ${ }^{3}$-that "slightly alkaline liquids were always more difficult to sterilise (by heat) than slightly acid liquids."

This difference came out strongest in my own experiments in the case of hay-infusion-the acid infusion invariably remaining barren after a few minutes' boiling, and the neutralised infusion invariably becoming fertile after a similar boiling. Accordingly I utilised hay-infusion to determine the cause of the difference in question. It could evidently only be due to one of two thingseither (I) the change of reaction enabled germs pre-existing in the infusion to survive the ebullition; or (2) the addition of the alkali exercised a positive infuence in exciting a de novo generation of organisms. To decide which of these two interpretations was the true one, an experiment was contrived in which the liquor potassa could be added to the infusion, not before, but after it had been boiled, and thereby rendered permanentlysterile. When adder in this way, I found that liquor potassæ had not any power to excite germination. The infusions invariably re mained barren when the alkali was added to them after they had been sterilised. I therefore concluded that the effect of the change of reaction consisted simply in enabling pre-existino germs to survive a brief ebullition. Dr. Bastian, in repeating this experiment in the case of urine, arrived at an opposite conclusion : he found that whether the alkali was added before or after ebullition he obtained the same result-the urine in both cases became fertile; and he concluded that the alkali had a positive power of promoting the origin of organisms in the urine.

This experiment, if properly performed, is obviously a crucial one, and it is recognised as such by Dr: Bastian. But two conditions are essential to the validity of the experiment. In the first place it must be ascertained beyond doubt that the boiled acid fluid has been really deprived of its germs-in other words, that the ebullition has been sufficiently prolonged to render it permanently barren; and secondly, that in adding the liquor potassæe due care is taken that no new germs are introduced at the same time. In repeating my experiment, Dr. Bastian ap. pears to have departed from my procerlure in two points, and he has thus possibly laid himself open to the two sources of fallacy just mentioned. In my own experiments, the acid infusion, after it had been boiled, was set aside in a warm place for a fortnight in order to test its sterility; and the liquor potassæe was not added to it until the lapse of time had satisfied me that it had been rendered permanently barren. In Dr. Bastian's experiments the liquor potassæ was added as soon as the vessels had cooled, so that he had no certainty that their contents would not have germinated without the addition of the alkali. ${ }^{4}$. In the second place, instead of heating the tubes containing the liquor potassæ (as I had done) to $15^{\circ} \mathrm{F}$, and thus ensuring the destruction of all germs contained in the air imprisoned therein

i " Note on the Influence of Liquor Potassæ and an Elevated Temperature on the Origin and Growth of Microphytes," by W. Roberts, M.D. Communicated by Prof. Tyndall, F.R.S., December 21, 1876 . 2 "Researches Illustrative of the Physico-Chemical Theory of Fermenta-
tion," \&c., read before the Royal Society, June 15,1876 .

3 Studies on Biogenesis. Phil. Trans. vol. lxiv. p. 457 .

4 It is not sufficient to rely in such a case on a central flask or retort. Each flask or retort should have its own individual sterility tested, because it is practically impossible to apply the heat exactly in the same degree in with the alkali, he contented himself with subjecting them for an inconsiderable period to the heat of boiling water.

Seeing these two possible sources of fallacy, I determined to repeat Dr. Bastian's experiments with urine, but taking care to avoid these defects. I proceeded as foilows:-

A flask with a longish neck was charged with an ounce of normal acid urine. The due quantity of liquor potassæ requisite to exactly neutralise this (as ascertained by previous trials) wite inclosed in a sealed glass tube drawn to a capillary portion at one end. The tube was then heated in oil up to $280^{\circ} \mathrm{F}$., and maintained at that temperature for fifteen minutes. The tube was then introduced into the body of the flask. The neck of the flask was next drawn to a narrow orifice; then the urine was boiled for five minutes, and the orifice sealed in ebullition. Ten such flasks were charged and treated in the same manner. They were then set aside in a warm place (from $70^{\circ} \mathrm{F}$, to $80^{\circ} \mathrm{F}$.) for a formight. At the end of this time the contents of the fiasks were found perfectly transparent; the urine was therefore assumed to be permanently sterilised. The liquor potassee was then liberated by shaking the tubes against the sides of the flasks and thus breaking their capillary points. The previously acid and barren urine was thus neutralised. The flasks were then placed in an incubator and maintained at a constant temperature of II $5^{\circ} \mathrm{F}$. At the end of two days it was found that the urine in each flask had deposited a sediment of earthy phosphates, but the surermatant liquor was perfectly transparent. The flasks were again placed in the incubator and maintained at a constant tempe ature of $122^{\circ} \mathrm{F}$. for three days. At the end of this period they were withdrawn and opened for examination. Not one of them showed the slightest evidence of living organisms; the supernatant liquor was perfectly transparent, and no Microphytes could be detected under the microscope. The precipitated phos. phate in some of the flasks presented a granular appearance, which might, by the unwary, be mistaken for Micrococci, but any such illusion was at once dissipated by adoing a drop of hydrochloric acid, which instantly dissolved the phosphate and restored the perfect transparency of the urine. This acid has no effect on the iurbidity caused by Microphytes.

These experiments therefore negative the conclusion that liquor potassa, or a temperature of $115^{\circ} \mathrm{F}$. to $I 22^{\circ} \mathrm{F}$., or both conditions combined, have the power of exciting the generation of organisms in sterilised urine.

The effect of elevated temperature was also tested in another way. I had by me twenty-nine preparations of fermentible liquids which had remained over from my previous expe:iments in 1873-74. These consisted of

$$
\begin{aligned}
& \text { I } 5 \text { alkalised hay-infusons, } \\
& 5 \text { pieces of boiled egg-albumen in water, } \\
& \text { I piece of turnip in water, } \\
& 2 \text { diluted ascitic fluid, } \\
& \text { I blood with water, } \\
& \text { I albuminous urine, } \\
& 4 \text { pieces of meat or fish in water. }
\end{aligned}
$$

These had all been sterilised by the heat of boiling water two or three years ago, and were contained in large bulbs with long necks. Ten of the hay-infusions were hermetically sealed; the rest were all open to the air, under the protection of a plug of cotton-wool. All possessed perfectly transuarent supernatant liquors, and showed no signs of containing organisms, nor of having undergone any fermentive or putrefactive changes.

These twenty-nine preparations were introduced into the inctibator, and maintained at a constant temperature of $\mathrm{II} 5^{\circ} \mathrm{F}$. for two days, and then at a temperature of $122^{\circ} \mathrm{F}$. for three days. At the end of this period not one of them showed any signs of fertility. The supernatant liquid in each bulb was quite transparent, and some of them, which were opened for microscopic examination, showed no traces of living organisms.

I can, horrever, fully confirm the statement of Dr. Bastian, that Bacteria, or certain kinds of them, grow and multiply freely in (unsterilised) urine, both acid and neutralised, when exposed to a temperature of $115^{\circ} \mathrm{F}$. to $122^{\circ} \mathrm{F}$.

The following is Prof. Tyndall's paper ${ }^{1}$ :-

The communication "On the Influence of Liquor Potassæa and an Elevated Temperature on the Origin and Growth of Microphytes," which, at Dr. Robexts's request, I have had the pleasure of presenting to the Royal Society, causes me to say earlier than I should otherwise have done, that the subject which has occu-

I "Note on the Deportment of Alkalized Urine," by Prof. Tyndail, F.R.S. Communicated December $2 \mathrm{I}, \mathrm{I} 876$. 